\title{
Papers
}

\section{Is electrodermal testing as effective as skin prick tests for diagnosing allergies? A double blind, randomised block design study}

George T Lewith, Julian N Kenyon, Jackie Broomfield, Philip Prescott, Jonathan Goddard, Stephen T Holgate

\begin{abstract}
Objective To evaluate whether electrodermal testing for environmental allergies can distinguish between volunteers who had previously reacted positively on skin prick tests for allergy to house dust mite or cat dander and volunteers who had reacted negatively to both allergens.

Design Double blind, randomised block design. Setting A general practice in southern England. Participants 15 volunteers who had a positive result and 15 volunteers who had a negative result on a previous skin prick test for allergy to house dust mite or cat dander.

Intervention Each participant was tested with 6 items by each of 3 operators of the Vegatest electrodermal testing device in 3 separate sessions (a total of 54 tests per participant). For each participant the 54 items comprised 18 samples each of house dust mite, cat dander, and distilled water, though these were randomly allocated among the operators in each session. A research nurse sat with the participant and operator in all sessions to ensure blinding and adherence to the protocol and to record the outcome of each test.

Outcome The presence or absence of an allergy according to the standard protocol for electrodermal testing.

Results All the non-atopic participants completed all 3 testing sessions (810 individual tests); 774 (95.5\%) of the individual tests conducted on the atopic participants complied with the testing protocol. The results of the electrodermal tests did not correlate with those of the skin prick tests. Electrodermal testing could not distinguish between atopic and non-atopic participants. No operator of the Vegatest device was better than any other, and no single participant's atopic status was consistently correctly diagnosed.

Conclusion Electrodermal testing cannot be used to diagnose environmental allergies.
\end{abstract}

\section{Introduction}

Unconventional allergy tests such as electrodermal testing are used widely in complementary and alterna-

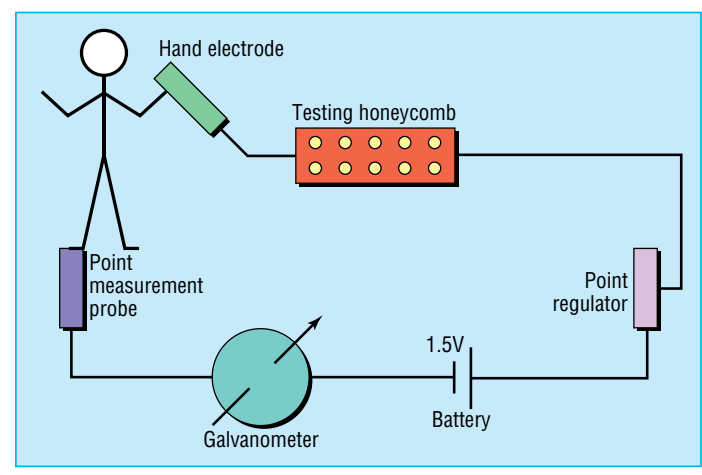

Fig 1 The electrical circuit used in the Vegatest

tive medicine. We wanted to compare the reliability of one common method of electrodermal testing for IgE dependent allergy, the Vegatest, with that of the gold standard for evaluating allergies, skin prick testing. The protocol for the Vegatest has been outlined elsewhere. ${ }^{1}$

Electrodermal testing was developed as an aid in prescribing homoeopathic remedies ${ }^{1}$ but is now widely used to assess an individual's allergic status to foods and aeroallergens. It is based on the observation that small changes in electrical impedance in the skin occur on an acupuncture point in response to substances placed in an electrical circuit (fig 1).

We estimate that more than 500 electrodermal devices are currently being used in the United Kingdom to assess sensitivity to potential allergens (see $B M J$ 's website for further details). We have no reliable information on the use of electrodermal testing in other countries or the extent to which it is used to detect food allergens rather than aeroallergens. Electrodermal testing for allergic and intolerance responses to dietary and environmental allergens-for which there are several different devices-has become increasingly popular, possibly because of its safety, non-invasive nature, and simplicity; therefore rigorous evaluation of electrodermal testing is important. The suggested mechanisms range from pressure changes over the acupuncture point that are produced unconsciously by the examiner to more objective mechanisms that are independent of the operator and are summarised as "quantum biology." 3
Department of
Medicine,
University of
Southampton,
Southampton
SO16 6YD
George T Lewith
honorary senior
research fellow
Jackie Broomfield
research nurse electrodermal tests
are available on the BMJ's website 
There have been several scientifically inadequate studies of electrodermal testing as a potential means of evaluating "allergy" ${ }^{4-9}$ Because the evidence base for using electrodermal testing to diagnose IgE dependent allergy is limited in quality and size, we undertook a clinical trial to test the hypothesis that electrodermal testing distinguishes between individuals who are sensitised to aeroallergens, as determined by skin prick tests, and non-atopic people.

\section{Methods}

\section{Selection of participants}

The study was carried out at a general practice, the Hythe Medical Centre, in Southampton. The volunteers were recruited through advertisements and from a departmental database. Volunteers were entered if they were aged between 18 and 65 but were excluded if they had any uncontrolled systemic disease, had taken part in any drug trial in the previous 30 days, had taken oral corticosteroids in the previous month or $\mathrm{H}_{1}$ antihistamines one week before electrodermal testing, or were pregnant. Those who reacted positively to a skin prick test for allergy to house dust mite or cat dander were entered as atopic. The skin prick test compares the effects of the test substance and a negative control when the skin is pricked through a drop of each substance. We defined a positive result as a wheal that 10 minutes after the puncture was $3 \mathrm{~mm}$ greater in diameter than that made by the control substance. The first 15 eligible volunteers were entered into each of the atopic and non-atopic groups. Skin prick testing, a method of evaluating allergic status that is reproducible and remains stable over a four month period, ${ }^{10}{ }^{11}$ was carried out under a standard protoco ${ }^{12}$ between 2 and 16 weeks before the electrodermal testing sessions. Ethical approval was obtained from the Southampton and south west Hampshire joint research ethics committee.

\section{The Vegatest protocol}

The operators were experienced electrodermal testers, each having used this procedure in a clinical setting for over 10 years. The four operators provided their own Vegatest machines (Vega Medizinische Geräte, Schiltach, Germany). The machines were set up in the standard manner for evaluating allergic responses, ${ }^{1}$ with four ampoules of homoeopathic epiphysis D26 and one ampoule of homoeopathic histamine D60 in the testing honeycomb (figure 1). The individual to be tested held a hand electrode, and the "Vega probe" was placed on the terminal acupuncture point on the lateral aspect of the third toe (the spleen/pancreas meridian). ${ }^{1}$ In each test the operator measured the skin impedance at this point as indicated in arbitrary units on the galvanometer while the patient held the hand electrode to complete the electrical circuit. Each time a new glass ampoule containing the experimental substance was placed in the Vegatest honeycomb, the operator again measured the skin impedance.

At the beginning of each testing session, each operator adjusted the baseline recording on the galvanometer so that the scale registered 100 units. The operator then placed an ampoule containing a toxic substance (the disorder control), such as mercury or paraquat, into the honeycomb in accordance with the standard Vegatest protocol. ${ }^{1}$ If the galvanometer reading was 60-70 units, the participant was diagnosed as "allergic," and the operator instructed the research assistant to note that the individual being tested was sensitive to the substance within the ampoule. If the galvanometer read 100 units, the classification "not allergic" was recorded. The magnitude of the readings is not considered to be proportional to the extent of allergic sensitisation. If the operator was unable to define the individual's allergic status on more than two ampoules tested at any testing session, that person's data were not included in the analysis.

Outcome, power calculation, and statistical analysis The measured outcome was the operator's assessment of the presence or absence of "allergy" for each of the ampoules tested on each individual participant. From previous reports, ${ }^{7-9}$ we expected that the sensitivity of the Vegatest would be about 0.7 and the false positive rate 0.15 , with little variation among participants. Assigning 12 people to each of the two groups would achieve a statistical power of $90 \%$ (a two sided normal test at a 5\% level of significance) even if the variance among participants was as high as 0.14 . Thus, to compensate for dropout we recruited 15 volunteers to each group. The statistician (PP) processed the data before breaking the randomisation codes.

\section{Intervention}

A pool of four operators performed the electrodermal tests over three fixed dates and a fourth flexible date for those who had difficulty attending one of the prebooked sessions. On each day three of the four operators attended the sessions at the clinic. At each session the participants were tested by each operator for their allergic reaction to six ampoules. Each participant was therefore evaluated for their response to 18 individual ampoules at each visit. The contents of the test ampoules were changed for each operator on each day. Each ampoule contained only one item, either an allergen extract (house dust mite or cat dander; Bayer Dome

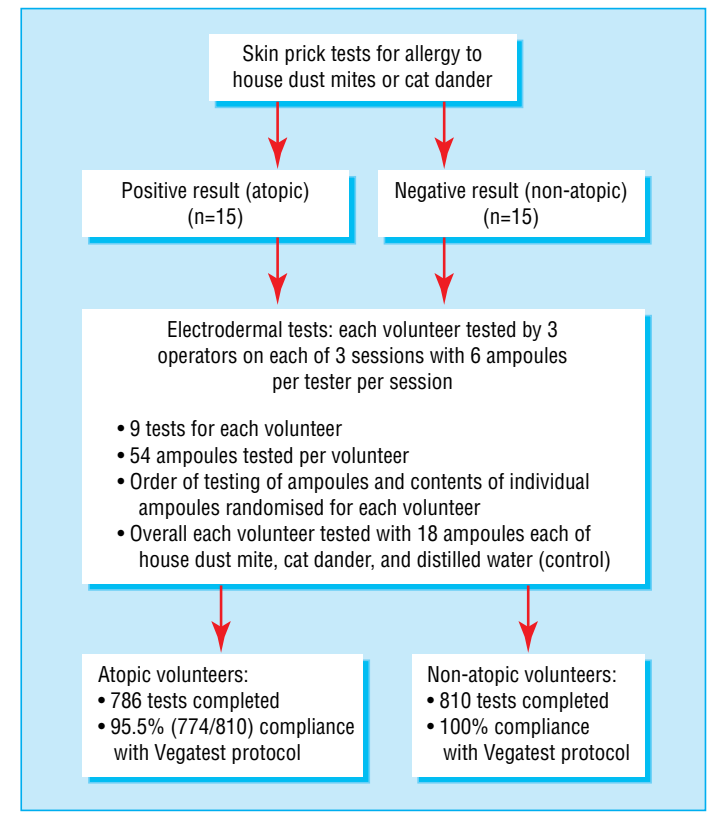

Fig 2 Study design 
Hollister Stier, Washington, DC) or a non-allergen (distilled water). Over all the tests in each session each participant was tested with six ampoules containing house dust mite extract, six of cat dander extract, and six of distilled water, but the contents of each individual set of six ampoules were randomised (fig 2).

\section{Assignment-blinding and randomisation}

The order of testing and the specific content of the ampoules was determined by random numbers generated by a computer. The order of presentation of the ampoules was randomised for each participant individually, and the order of testing was randomised in blocks of six ampoules. The sets of ampoules were made up at an independent homoeopathic dispensary (the Centre for the Study of Complementary Medicine, Southampton) with numerical codes on each of the bottles. The ampoules containing the two allergens could not be distinguished from one another or from the distilled water. To ensure complete blinding the trial manager was not present at any of the individual testing sessions. A research assistant stayed with the operators throughout each session to ensure that they did not question the participants on their medical history. The research assistant also placed each ampoule to be tested into the Vegatest honeycomb and recorded the operator's identification of the participant as either allergic or not allergic. The research assistant then returned the set of six ampoules to the trial manager at the end of each clinical session.

\section{Results}

A total of 1596 individual tests were completed. All 15 non-atopic participants completed all the tests and complied with the testing protocol (810 tests). The atopic participants completed 786 tests, of which 774 complied with the protocol (figure 2). The percentages of "allergic" and "non-allergic" participants as determined by the operators are shown in table 1 . Overall the operators reported a positive (allergic) response (a reading of 60-70 units of the scale) in a quarter of the tests and a negative (non-allergic) response in nearly three quarters; they were unable to assess $1.0 \%$ of the responses as either allergic or non-allergic.

The Vegatest readings from the atopic group did not differ significantly from those of the non-atopic groups. The mean percentage of Vegatest results that accorded with the results of the skin prick tests was normally distributed, and the Vegatest was unable to differentiate between atopic and non-atopic groups for each of the allergens: $24 \%$ of atopic participants versus $22 \%$ of nonatopic participants were diagnosed by the Vegatest operators as being sensitive to cat dander; $28 \%$ versus $29 \%$ to house dust mite, and $26 \%$ versus $23 \%$ to distilled water. No individual participant's allergic status as determined by skin prick testing was consistently correctly diagnosed by all the Vegatest operators.

We also analysed each operator's results for each substance tested in both groups of participants and found no significant differences from the overall patterns. The operators' overall results are shown in table 2 . There was no difference in reliability among the operators, no significant differences in the accuracy of individual operators, and no significant positive or negative correlation between the results of the Vegatest and
Table 1 Allergic status of atopic or non-atopic* participants as reported by operators of the Vegatest. Figures are numbers (percentages for each substance)

\begin{tabular}{lcccc} 
& “Allergic" & "Non-allergic” & $\begin{array}{c}\text { Allergic status not } \\
\text { determined }\end{array}$ & Total \\
\hline Atopic volunteers: & & & & \\
\hline Cat dander & $61(24)$ & $195(76)$ & $2(1)$ & 258 \\
\hline House dust mite & $73(28)$ & $183(71)$ & $3(1)$ & 259 \\
\hline Distilled water & $66(26)$ & $188(73)$ & $3(1)$ & 257 \\
\hline Total & $200(26)$ & $566(73)$ & $8(1)$ & 774 \\
\hline Non-atopic volunteers: & & & & \\
\hline Cat dander & $59(22)$ & $209(78)$ & $1(<1)$ & 269 \\
\hline House dust mite & $78(29)$ & $191(70)$ & $2(1)$ & 271 \\
\hline Distilled water & $63(23)$ & $202(75)$ & $5(2)$ & 270 \\
\hline Total & $200(25)$ & $602(74)$ & $8(1)$ & 810
\end{tabular}

*Determined previously by skin prick tests.

those of the skin prick test for any operator (data not shown). Analysis of false positive and false negative data did not show any significant differences among the operators or any significant correlation between the results of the Vegatest and those of the skin prick test.

\section{Discussion}

This double blind, randomised block design study, which comprised over 1500 observations, showed that electrodermal testing could not distinguish atopic from non-atopic individuals. No operator was more reliable than any other, and no participants were consistently correctly diagnosed.

As IgE dependent food allergy-for example, to milk, eggs, or nuts-has the same pathophysiological basis as mucosal responses to aeroallergens, we conclude that the Vegatest is an inappropriate tool for diagnosing any form of immediate hypersensitivity. However, we recognise that electrodermal testing, when used to assess "antigens" in a non-blinded manner, is usually used to evaluate food intolerance rather than to diagnose allergies in the traditional sense of the term. ${ }^{8}$ We did not investigate food intolerance, partly because there is no universally recognised conventional test for food intolerance against which to evaluate electrodermal testing. Nevertheless, it should be possible to design an appropriate trial to study the diagnostic usefulness of the Vegatest for food intolerance reported by patients, although it is difficult to envisage a unifying mechanism to explain how the test would be able to detect this heterogeneous group of disorders. Furthermore, we recognise that electrodermal testing was developed to aid the prescription of homoeopathic and herbal remedies and not primarily as an allergy test; we made no attempt to evaluate these claims. ${ }^{1}$ The phenomena involved in electrodermal testing may make it difficult to evaluate in an entirely blind manner, as the person conducting the test may be an important part of the process. ${ }^{23}$ Such issues can be addressed by appropriate randomised, controlled

Table 2 Overall results of tests for the Vegatest operators.

Figures are numbers (percentages)

\begin{tabular}{lccc} 
& "Allergic" & “Non-allergic” & $\begin{array}{c}\text { Allergic status } \\
\text { not determined }\end{array}$ \\
\hline Tester 1 & $214(38)$ & $350(62)$ & 0 \\
\hline Tester 2 & $128(27)$ & $332(69)$ & $20(4)$ \\
\hline Testers 3 and 4 & $58(11)$ & $486(88)$ & $8(1)$ \\
\hline Total & $400(25)$ & $1168(73)$ & $28(2)$ \\
\hline
\end{tabular}




\section{What is already known on this topic}

Unconventional testing for allergy, such as electrodermal testing, is widely available

Few clinical trials have evaluated unconventional testing

\section{What this study adds}

Electrodermal testing cannot distinguish between atopic and non-atopic individuals as previously determined by skin prick tests

trials. We conclude that electrodermal testing cannot diagnose allergy to common aeroallergens such as cat dander and house dust mite-allergens that have a strong association with atopic respiratory disorders such as asthma, rhinitis, eczema, and conjunctivitis.

We thank Jackie Burnham for her help in preparing the manuscript, all the volunteers and the operators who gave up their time so willingly, and the doctors and practice manager at Hythe Medical Centre who made space and time available to us.

Contributors: GTL, JNK, and STH conceived the study, developed the protocol, and obtained funding. JB was the trial manager. JG was responsible for the statistical design and randomisation, and $\mathrm{PP}$ for the analysis. All authors were jointly involved in writing the paper. STH will act as guarantor.
Funding: The study was supported by funding from Vega Grieshaber, the Asthma Allergy and Inflammation Research Trust, and the Foundation for Integrated Medicine.

Competing interests: GTL and JNK formerly had interests in a company involved in the distribution of Vega Grieshaber products and both have received fees for speaking at a seminar on the Vegatest.

1 Kenyon J, ed. Short manual of the Vegatest method. Schiltach, Germany: Vega Grieshaber, 1981.

2 Van Wijk R, Wilegart FAC. Homoeopathic remedies and pressure induced changes and the galvanic resistance of the skin. Utrecht: State University of Utrecht, Research Unit for Complementary Medicine, 1989.

3 Ho MW. The rainbow and the worm-the physics of organisms. Singapore and London: World Scientific, 1993.

4 Tsuei J and Madill P. A food allergy study using the EAV acupuncture technique. Am J Acupunct 1984;12:105-16.

5 Fuller Royal F, Fuller Royal D. Scientific support for electrodiagnosis. $\mathrm{Br}$ Homoeopathic J 1991;18:166-78.

6 Fox A. Determination of neutralisation point for allergic hypersensitivity. Br Homoeopathic J 1987;76:230-4

7 Ali M. Correlation of IgE antibodies with specificity for pollen and mould allergy changes in electrodermal skin responses following exposure to allergens. Am J Clin Pathol 1989;91:357-9.

8 Krop J, Swiesczek J, Wood A. Comparison of ecological testing with the Vegatest method in identifying sensitivities to chemicals, foods and inhalants. Am J Acupunct 1985;13:253-9.

9 Krop J, Lewith, G, Gziut W, Radulescu C. A double-blind, randomised, controlled investigation of electrodermal testing in the diagnosis of allergies. J Altern Complement Med 1997;3:241-8.

10 Dreborg A, Frew A. Allergen standardisation and skin tests. EAACI '93 subcommittee on skin tests. Allergy 1993;14:48-82.

11 Gallant SP, Maibach HI. Reproducibility of allergy epicutaneous techniques. J Allergy Clin Immunol 1973;51:245-50.

12 Basomba A. Evaluation of changes in skin sensitivity by means of skin tests. EAACI'93 subcommittee on skin tests. Allergy 1993;14:71-5.

(Accepted 20 October 2000)

\section{Treatment for the premenstrual syndrome with agnus castus fruit extract: prospective, randomised, placebo controlled study}

R Schellenberg for the study group

Institute for Health Care and Science, 35625 Hüttenberg Germany

R Schellenberg senior consultant

Correspondence to: rued.schellenberg. med@t-online.de

BMJ 2001;322:134-7

\section{Abstract}

Objectives To compare the efficacy and tolerability of agnus castus fruit (Vitex agnus castus L extract Ze 440) with placebo for women with the premenstrual syndrome.

Design Randomised, double blind, placebo controlled, parallel group comparison over three menstrual cycles.

Setting General medicine community clinics. Participants 178 women were screened and 170 were evaluated (active 86; placebo 84). Mean age was 36 years, mean cycle length was 28 days, mean duration of menses was 4.5 days.

Interventions Agnus castus (dry extract tablets) one tablet daily or matching placebo, given for three consecutive cycles.

Main outcome measures Main efficacy variable: change from baseline to end point (end of third cycle) in women's self assessment of irritability, mood alteration, anger, headache, breast fullness, and other menstrual symptoms including bloating. Secondary efficacy variables: changes in clinical global impression (severity of condition, global improvement, and risk or benefit) and responder rate (50\% reduction in symptoms).
Results Improvement in the main variable was greater in the active group compared with placebo group $(\mathrm{P}<0.001)$. Analysis of the secondary variables showed significant $(\mathrm{P}<0.001)$ superiority of active treatment in each of the three global impression items. Responder rates were $52 \%$ and $24 \%$ for active and placebo, respectively. Seven women reported mild adverse events (four active; three placebo), none of which caused discontinuation of treatment.

Conclusions Dry extract of agnus castus fruit is an effective and well tolerated treatment for the relief of symptoms of the premenstrual syndrome.

\section{Introduction}

The premenstrual syndrome is a complex combination of psychological symptoms, including irritability, aggression, tension, anxiety, and depression, and somatic changes such as fluid retention, breast tenderness, headache, feeling of bloating, and weight increase. ${ }^{1}$ Women are affected irrespective of socioeconomic status, race, or cultural background, and family clusters are well documented. ${ }^{23}$ The causes of the premenstrual syndrome have not been clearly elucidated ${ }^{4}$ but have been attributed to hormonal change, neurotransmitters, 